\title{
LS-SVM Assessment of Weapon System Development Risk Based on SPA
}

\author{
Chunlan Wang ${ }^{1, \text { a }}$, Xusheng Gan ${ }^{2, b}$, Shenghou $\mathrm{Li}^{2, \mathrm{c}}$ \\ ${ }^{1}$ Xijing College, Xi’an, Shaanxi, 710123, China \\ ${ }^{2}$ Air Traffic Control and Navigation College, Air Force Engineering University, Xi’an, Shaanxi, 710051, \\ China \\ awangcl888@163.com, banxusheng123@163.com, ㄷish58@163.com
}

Keywords: Set pair analysis; LS-SVM; Weapon system development; Risk assessment

\begin{abstract}
To effectively prevent and development risk of weapon weapon system, a comprehensive assessment method based on Set Pair Analysis (SPA) theory and Least Square Support Vector Machine (LS-SVM) method is proposed for weapon weapon system development. firstly, on the basis of built weapon weapon system development risk assessment index system, the concept of connection degree and set pair in SPA theory is introduced to construct the training samples and test samples.Then through the obtained samples LS-SVM is trained and tested to get the assessment model and give the assessment result. The example shows that, the proposed method has many advantages in simple implement, combining qualitative and quantitative analysis, and easy understanding.
\end{abstract}

\section{Set pair analysis theory}

Set Pair Analysis (SPA) method is a systematic theory and method proposed by Zhao Keqin in 1989 to deal with the problem of uncertainty. The core idea is to first construct the set pair of two related sets in an uncertain system, then conduct the analysis on identity, difference, and antagonism for characteristics of set pair, finally compared with traditional fuzzy comprehensive evaluation method it is good at dealing with fuzzy information. The gray comprehensive assessment method is good at dealing with gray information [1][2]. The advantage of SPA method is that it can uniformly deal with the uncertainty caused by fuzzy, gray, random, missing information. At present, this method has been applied in many fields such as aerospace, earth environment, geological disasters and military defense.

Based on this, the comprehensive assessment steps of the SPA can be given as follows:

1) Select the index (or factor) domain of the assessment object;

Suppose that the system to be assessed has $n$ indices, and can be expressed as $X=\left(X_{1}, X_{2}, \cdots, X_{n}\right)$.

2) Determine the domain of assessment level;

According to the value range of $\mu$, the domain of assessment level is divided following the certain principles.

3) Calculate the weight of the indices;

The weight reflects the coefficients of the importance of each index in the assessment system, usually determined by expert scoring or AHP.

4) Calculate the result of comprehensive assessment.

When considering the weight, the following formula can be used to calculate the result of the comprehensive assessment 


$$
\mu=W \cdot R \cdot E=\left[\omega_{1}, \omega_{2}, \cdots, \omega_{n}\right]\left[\begin{array}{ccc}
a_{1} & b_{1} & c_{1} \\
a_{2} & b_{2} & c_{2} \\
\cdots & \ldots & \cdots \\
a_{n} & b_{n} & b_{n}
\end{array}\right]\left[\begin{array}{c}
1 \\
i \\
j
\end{array}\right]
$$

where $W$ is the expert weight matrix; $R$ is the identity, difference, and antagonism matrix; $E$ is the relation component matrix.

\section{Assessment Process of Weapon system Development Risk}

In fact, the proposed LS-SVM [3][4] assessment process of weapon system development risk based on SPA analysis in the paper, is to firstly obtain the sample data through SPA analysis on the basis of Index system of weapon system development risk assessment as shown in Figure 1, and then train the weapon system development risk assessment model by LS-SVM learning to get the risk assessment results for the new sample. Usually, in order to make the model acquire more knowledge, the new validated assessment results need to be used as new samples that should be learned again. As the number of learning samples increases, the results of LS-SVM will be more accurate. The proposed weapon system development risk assessment process is shown in Figure 2.

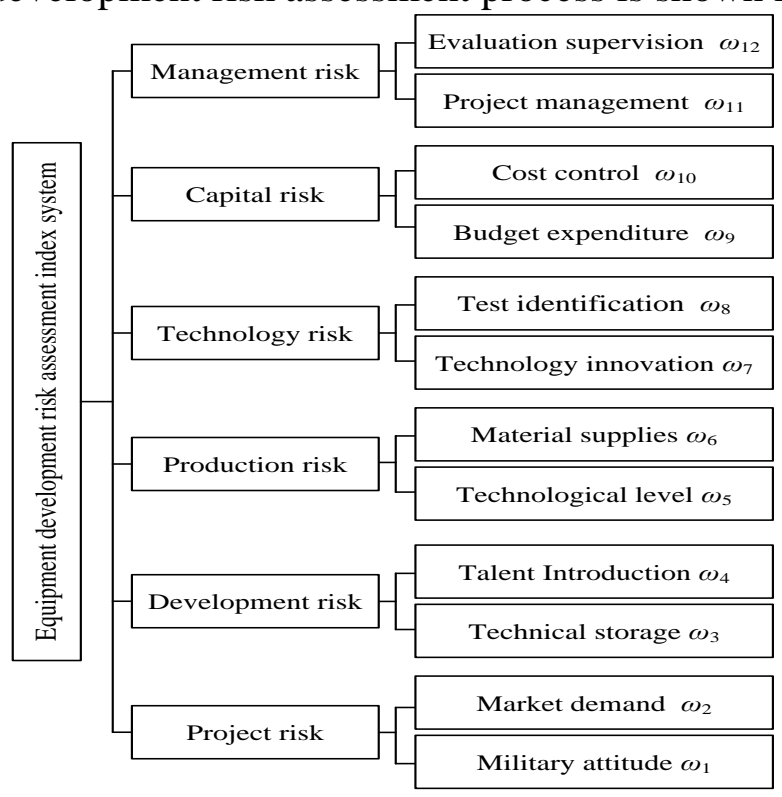

Figure 1 Index system of weapon system development risk assessment

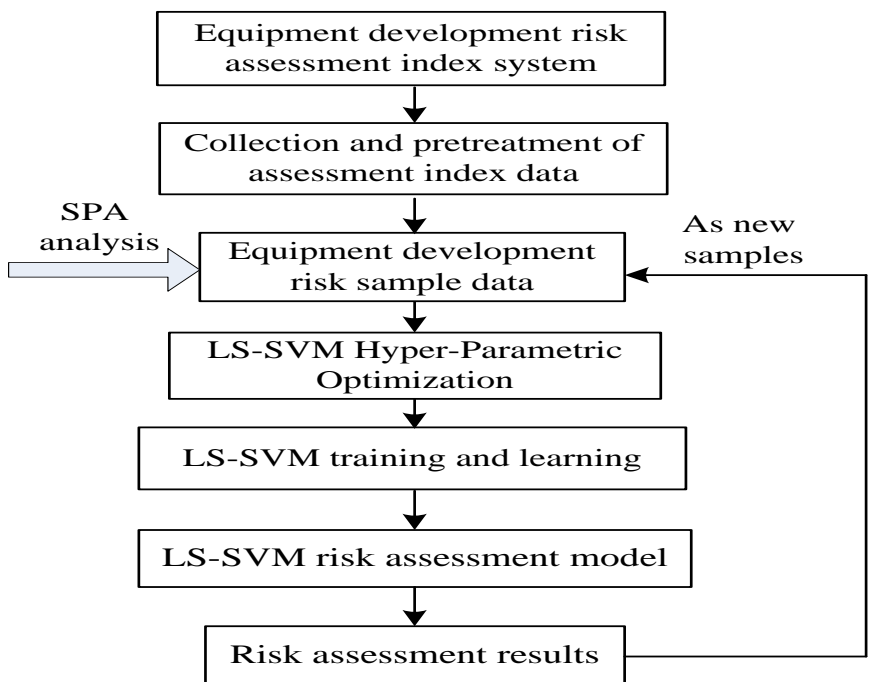

Figure 2 Weapon system development risk assessment process 
According to the process of weapon system development and its risk characteristics, the comprehensive assessment level of weapon system development risk can be divided into 5 levels, namely I, II, III, VI and V. Level I: Very high risk level, it cannot continue to develop. Level II: High risk level, major measures need to be taken in a targeted manner to eliminate risks; Level III: Medium risk level, some precautions should be taken; Level IV: Low risk level, There are hidden dangers in the system, and need timely rectification and solution; Level V: Best risk level, the weapon system research and development is in good condition; At the same time, each assessment index is also divided into 5 levels.

An index and its level standard of weapon system development risk assessment were listed as two sets respectively, constituting a set pair. If the index value is within this assessment level range, it is considered to be the identity; if the index value is in the adjacent assessment level, it is considered as the difference; and if the index value is in the separated assessment level, it is considered to be the antagonism. Thus, in order to quantify the connection degree, for the index $U$, the connection degree function can be constructed as follows:

$$
\begin{gathered}
\mu_{i 1}=\left\{\begin{array}{ll}
1 & x \in\left[U_{1}, U_{0}\right) \\
\frac{x-U_{2}}{U_{1}-U_{2}} & x \in\left[U_{2}, U_{1}\right) \\
0 & x \in\left[0, U_{2}\right)
\end{array} \quad \mu_{i 2}=\left\{\begin{array}{ll}
\frac{U_{0}-x}{U_{0}-U_{1}} & x \in\left[U_{1}, U_{0}\right) \\
\frac{x-U_{3}}{U_{2}-U_{3}} & x \in\left[U_{3}, U_{2}\right) \\
0 & x \in\left[0, U_{3}\right)
\end{array} \quad \mu_{i 3}= \begin{cases}0 & x \in\left(0, U_{4}\right] \cup\left[U_{1}, U_{0}\right) \\
\frac{U_{1}-x}{U_{1}-U_{2}} & x \in\left[U_{2}, U_{1}\right) \\
\frac{x-U_{4}}{U_{3}-U_{4}} & x \in\left[U_{4}, U_{3}\right)\end{cases} \right.\right. \\
\mu_{i 4}=\left\{\begin{array}{ll}
0 & x \in\left(0, U_{5}\right) U\left[U_{2}, U_{0}\right) \\
\frac{U_{2}-x}{U_{2}-U_{3}} & x \in\left(U_{3}, U_{2}\right] \\
\frac{1}{x-U_{5}} & x \in\left(U_{4}, U_{3}\right]
\end{array} \quad \mu_{i 5}= \begin{cases}0 & x \in\left(0, U_{5}\right] \cup\left[U_{3}, U_{0}\right) \\
\frac{U_{3}-x}{U_{3}-U_{4}} & x \in\left[U_{4}, U_{3}\right) \\
1 & x \in\left[U_{5}, U_{4}\right)\end{cases} \right.
\end{gathered}
$$

where $\mu_{i 1}, \mu_{i 2}, \mu_{i 3}, \mu_{i 4}, \mu_{i 5}$ are the connection degree when the assessment index value is in Level $\mathrm{V}$, IV, III, II, and I respectively. $U_{0} \sim U_{5}$ are the limitation value of each level of the assessment index respectively; $x$ is the actual value of weapon system development risk assessment index; $i$ is the serial number of different indices. Calculate the connection degree $\mu_{i j}(j=1,2,3,4,5)$ of the $i$-th index under different assessment levels. The total connection degree can be calculated by following formula

$$
\mu_{j}=\sum_{i=1}^{n} \mu_{i j} \omega_{i}
$$

Where $\mu_{j}$ is the total connection degree of each index to the $j$-th level; $\omega_{j}$ is the weight of each index. The level with the highest connection degree is selected as the final risk assessment level.

\section{Validation example}

In the paper, the risk assessment of a ground support weapon system development project for Air Force is taken as the research background of case analysis. The total assessment process is divided into 2 parts. The first part: SPA is used to assess the weapon system development risk and determine the training and test samples for LS-SVM. The second part: On the basis of SPA assessment, LS-SVM assessment model of weapon system development risk is trained and is tested. 
From the assessment results of weapon system development risk, it can be seen that the assessment results of SPA and LS-SVM are generally the same. However, compared with the former, LS-SVM has more advantages, this mainly reflects in 3 aspects: First, LS-SVM takes a "black box" thinking to modeling, namely it can find out the relationship between input variables and output variables through continuous learning and memory. we only need to input each index data into the trained LS-SVM network. It performs the deduction and reasoning according to the stored knowledge in the network, drawing the assessment results. Although the calculation of LS-SVM is more complex than that of SPA, however, with the help of computer, it can simplify the assessment process and save the assessment time. Second, in the training process, it is not necessary to determine the weight according to the expert knowledge as SPA does in advance. LS-SVM can automatically adjust the weight of each index according to the training sample, and can solve the dynamic weight change question of each index weight that determines the future state or trend according to the historical and current information. Third, LS-SVM assessment model has a strong associative memory function. Even for new samples that are not involved in training, a more reasonable risk assessment result can be obtained based on the learning of historical information, whereas SPA is heavily dependent on Expert knowledge. In the paper, SPA and LS-SVM are combined to carry out weapon system development risk assessment. That is to say, the assessment results SPA are used to construct samples to train and test LS-SVM weapon system development risk assessment model, which not only takes advantage of LS-SVM assessment but also effectively solves the problem of samples source for LS-SVM training. As the assessment process in Figure 2, after LS-SVM model training based on SPA sample is completed, we can carry out multiple weapon system development risk assessment. Unless considering the needs of model updating (adding new samples), otherwise, LS-SVM models need not be trained on every assessment.

Table 1 Assessment results of LS-SVM under different initial parameters

\begin{tabular}{|c|c|c|c|c|}
\hline initial parameter & \multicolumn{2}{|c|}{ No.11 sample } & \multicolumn{2}{c|}{ No.12 sample } \\
\hline$C=100, \sigma^{2}=20$ & 2.9841 & III (3) & 4.1356 & IV (4) \\
\hline$C=50, \quad \sigma^{2}=10$ & 3.0246 & III (3) & 4.0512 & IV (4) \\
\hline$C=200, \quad \sigma^{2}=5$ & 3.1729 & III (3) & 3.8925 & IV (4) \\
\hline$C=10, \quad \sigma^{2}=10$ & 2.9548 & III (3) & 4.2121 & IV (4) \\
\hline
\end{tabular}

\section{Conclusions}

An integirated assessment method based on SPA and LS-SVM is proposed for weapon system development risk. In this assessment method, SPA is used to assess the weapon system development risk and determine the training and test samples for LS-SVM, and then LS-SVM assessment model of weapon system development risk is trained and validated. The simulation results show that the proposed method gives a satisfactory risk assessment result for weapon system development.

\section{References}

[1] S. C. Tian, L. Wang, H. X. Li. Application of risk evaluation in coalmine gas hazard based on model of SPA. Journal of Safety and Environment, 6(6), (2006), 103-106.

[2] H. J. Xu, B. Gong, D. S. Li. Risk assessment of coal mine based on set pair analysis. Journal of Safety Science and Technology, 8(6), (2012), 86-90.

[3] J. A. K. Suykens, J. Vandewalle. Least squares support vector machine classifiers. Neural Processing Letters, 9(3), (1999), 293-300.

[4] X. H. Han, S. H. Du, J. Su. Determination method of electric shock current based on parameter-optimized least squares support vector machine. Transations of the Chinese Society of Agricultural Enineering, 30(23), (2014), 238-244. 